\title{
Design and operation of a field telescope for cosmic ray geophysical tomography
}

\author{
N. Lesparre ${ }^{1, *}$, J. Marteau ${ }^{2}$, Y. Déclais ${ }^{2}$, D. Gibert ${ }^{1}$, B. Carlus ${ }^{2}$, F. Nicollin ${ }^{3}$, and B. Kergosien ${ }^{3}$ \\ ${ }^{1}$ Institut de Physique du Globe de Paris, UMR7154, CNRS, Sorbonne Paris Cité, Paris, France \\ ${ }^{2}$ Institut de Physique Nucléaire de Lyon, UMR5822, CNRS IN2P3 - Université Lyon 1, Lyon, France \\ ${ }^{3}$ Géosciences Rennes, UMR6118, CNRS - Université Rennes 1, Bât. 15 Campus de Beaulieu, 35042 Rennes cedex, France \\ *now at: Systems and Computer Engineering, Carleton University, Ottawa, Canada
}

Correspondence to: N. Lesparre (lesparre@ipgp.fr)

Received: 18 November 2011 - Published in Geosci. Instrum. Method. Data Syst. Discuss.: 19 December 2011 Revised: 22 March 2012 - Accepted: 4 April 2012 - Published: 20 April 2012

\begin{abstract}
The cosmic ray muon tomography gives an access to the density structure of geological targets. In the present article we describe a muon telescope adapted to harsh environmental conditions. In particular the design optimizes the total weight and power consumption to ease the deployment and increase the autonomy of the detector. The muon telescopes consist of at least two scintillator detection matrices readout by photosensors via optical fibres. Two photosensor options have been studied. The baseline option foresees one multianode photomultiplier (MAPM) per matrix. A second option using one multipixel photon counter (MPPC) per bar is under development. The readout electronics and data acquisition system developed for both options are detailed. We present a first data set acquired in open-sky conditions compared with the muon flux detected across geological objects.
\end{abstract}

\section{Introduction}

The abundance and the large energy range of the atmospheric muons (Gaisser and Stanev, 2008), combined with the fact that the muons interact only weakly with matter, make them an appropriate probe for attempting tomographies of kilometer scale geological objects (Barrett et al., 1952). The attenuation of the flux of muons propagating through geological layers (Gaisser and Stanev, 2008) provides information on the averaged density along the muon trajectories inside the rock volume (Nagamine, 2003).

Muon radiography has first been applied in the seventies for archaeological investigations in the Egyptian Chephren pyramid (Alvarez et al., 1970). Later, theoretical considerations on the feasibility of muon radiography in mining engineering appeared in the geophysical literature (Malmqvist et al., 1979). The idea of muon radiography in volcanic studies first appeared in 1995 (Nagamine, 1995; Nagamine et al., 1995) and further development in a detector design and a data analysis method has been worked out (Tanaka et al., 2003). The first muon radiography of a volcano was obtained by Tanaka et al. (2005). These pioneering studies, soon followed by further experiments, aimed at detecting both spatial and temporal variations of density inside volcanoes (Tanaka et al., 2009, 2010 and reference therein).

We develop cosmic ray telescopes designed for imaging geological targets with a thickness of rock between 50 and $1500 \mathrm{~m}$. The detection time from each measurement location has to remain less than six months, to construct in a few years a tomography of the object. This method allows the detection of objects with a spatial resolution between 10 and $40 \mathrm{~m}$, for telescopes with an angular resolution of about $0.1 \mathrm{rad}$. The density variations detectable with such a method could be as low as $3 \%$, depending on the rock opacity as well as on the respective sizes of the target and the heterogeneities (Lesparre et al., 2010). Some first acquisitions have to be realised in open sky conditions to control the telescopes' bars efficiency and calibrate their set-up (Lesparre et al., 2012).

The feasibility of muon radiography is now well established, and the present challenge is to perform full 3-D tomography by placing muon telescopes all around the geological target to obtain the multi-directional ray coverage necessary to solve the inverse problem of 3-D tomography 
(Mohammad-Djafari and Dinten, 2008). A first study of this kind was recently performed by Tanaka et al. (2010). This objective poses a number of practical problems because many geological targets of interest are explosive volcanoes with unstable steep topography, located in remote tropical areas with harsh environmental conditions (Gibert et al., 2010; Marteau et al., 2011).

In the present paper, we describe cosmic ray telescopes designed for such field environment. These telescopes are intended to operate on tropical volcanoes like La Soufrière of Guadeloupe with a relative humidity higher than $95 \%$, heavy rains and strong winds. There, most telescope locations are far from roads and power lines, and the total weight and power consumption must be low enough to allow both helicopter hauling and solar panel powering. In this paper we present the detection system and the data acquisition chain in Sects. 2 and 3, the mechanical frame and the field conditioning equipment (wireless links, solar panels) in Sect. 4 and the telescope response calibration for open sky conditions in Sect. 5. Finally, we end with first field measurements.

\section{Detection system}

\subsection{Scintillator bars}

Different detection techniques are available: emulsions (Tanaka et al., 2007), resistive plate chambers (De Asmundis et al., 2007), micromegas (Giomataris et al., 2006), scintillators (Pla-Dalmau et al., 2001). We retained the scintillator bars option to build the detection matrices since plastic scintillators are known to be robust, light, low-cost detectors particularly well suited to field conditions and actually used for most studies on volcanoes (Tanaka et al., 2005; Gibert et al., 2010; Marteau et al., 2011). Furthermore, scintillators allow the use of the compact OPERA data acquisition system described later in the present paper.

The scintillator bars have a rectangular cross-section of $5 \times 1 \mathrm{~cm}^{2}$ and are co-extruded with a $\mathrm{TiO}_{2}$ reflective coating and a $1.5 \mathrm{~mm}$ diameter central duct to host an optical fibre for light collection (Pla-Dalmau et al., 2001). The photons produced in a scintillator bar are indeed collected by a wavelength shifting (WLS) optical fibre (Bicron BCF 91A MC) glued with an optical cement (Bicron BC-600) in the central duct of the bar. The WLS fibres are only polished at one end since their length $(0.8,1.2$ or $1.6 \mathrm{~m})$ is short compared to the light attenuation length (approximatively $10 \mathrm{~m}$ ). This extremity of the fibre is glued in an optical plug designed to receive either a clear optic fibre (Bicron BCF-98 MC) or a Multi-Pixel Photon Counter (see Sect. 2.3).

\subsection{Matrices set up}

The scintillator bars described above are aligned in the orthogonal $\mathrm{X}$ and $\mathrm{Y}$ directions to form a checker-board arrangement constituting a matrix whose $5 \times 5 \mathrm{~cm}^{2}$ pixels are

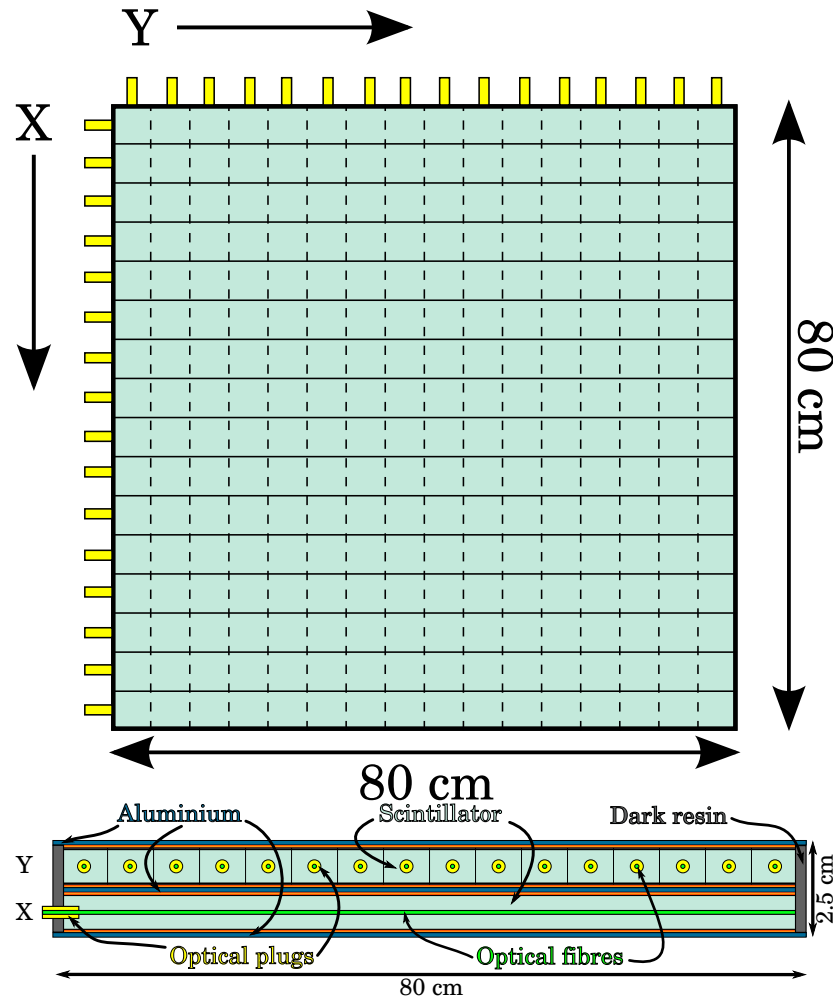

Fig. 1. Top: schematic view of a matrix with $16 \times 16$ pixels, an area of $0.8 \times 0.8=0.64 \mathrm{~m}^{2}$ and one optical plug at one extremity of each bar. Bottom: cross-section view.

defined as the intersections of the $\mathrm{X}$ and $\mathrm{Y}$ bars (Fig. 1). We built such matrices with $16 \times 16$ and $24 \times 32$ pixels, respectively. The smaller matrices with a size of $0.8 \times 0.8=$ $0.64 \mathrm{~m}^{2}$ were the first built to validate the manufacturing procedure. The larger matrices offer a larger detection area which reduces the acquisition time for a given angular resolution (Lesparre et al., 2010).

The two $\mathrm{X}$ and $\mathrm{Y}$ scintillator layers are encapsulated by $1.5 \mathrm{~mm}$ thick anodised aluminium plates. The four edges of the matrix are filled with a black polyurethane resin (Axson RE-11820-95) to form a compact self-supporting rigid board protected against light and humidity (Fig. 1). The three aluminium sheets are grounded with a common copper adhesive tape to avoid capacitor phenomena. The total thickness of a matrix is of about $26 \mathrm{~mm}$ for a weight of $25 \mathrm{~kg}$ for a $16 \times 16$ matrix. One telescope contains at least two matrices (Fig. 2) to define the trajectory of a detected particle from the pixels fired on each matrix. The total aperture angle and the angular resolution of the telescope may be adjusted by changing the distance between the matrices. For instance, for distances of 90,115 and $150 \mathrm{~cm}$, the total aperture angular are of $1.5,1.2$ and $1.0 \mathrm{rad}$ for angular resolutions of 111,87 and $66 \mathrm{mrad}$, respectively. 


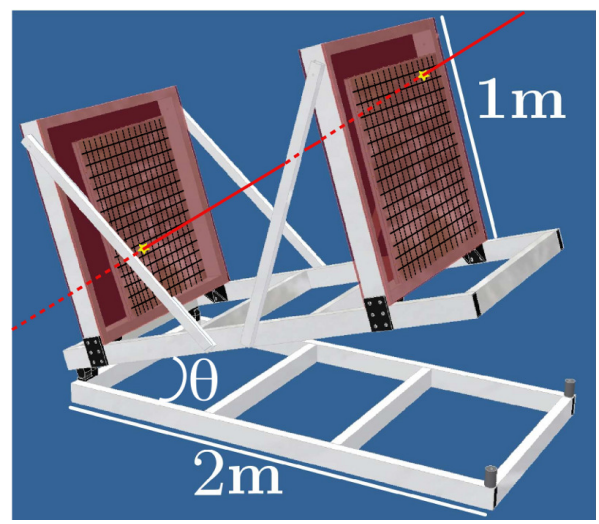

Fig. 2. Schematic view of a muon telescope equipped with two matrices with $16 \times 16$ pixels.

\subsection{Photomultipliers}

Two types of photomultipliers may be adapted to the matrices. The first type is a 64-channels Multi-Anode PhotoMultiplier (MAPM) connected to the matrix bars through clear optical fibres, and the second type is for Multi-Pixel Photon Counter (MPPC or Silicon PM, SiPM) directly connected onto the optical plugs of the scintillator bars.

MAPM requires clear optical fibres connection to guide the light from the scintillator bars to the sensor's entrance window. Depending on the size of the matrix, up to $64 \mathrm{fi}-$ bres may be glued in a dedicated cookie which insures the fibre-to-pixel alignment (Fig. 3). The clear fibres are covered with a black coat to insure light tightness. The cookie contains a light diffusing system with a LED to calibrate in-situ the MAPM response. Although the use of optical grease (Nuclear Enterprise NE588) increases the coupling by up to $20 \%$, the large time-variations and larger gain nonuniformity which have been observed lead us to prefer a direct contact between the MAPM external window and the cookie.

Our first telescopes are readout with MAPMs (Hamamatsu H8804-mod1), compatible with the data acquisition system already developed for the OPERA Target Tracker experiment (Adam et al., 2007). The MAPM has a time resolution between 2 and $5 \mathrm{~ns}$. The gain and pedestal variations measured on field are lower than $10 \%$ and $1 \%$, respectively.

The MPPCs (Hamamatsu S10362-11 series ref. 050C and 100C) have very attractive performances in terms of low light level detection, single photon sensitivity and photon number resolution power. Their form factor and ceramic packaging are optimal for the coupling to a scintillator. They fit into the optical plugs originally designed for the clear fibres used in the MAPM option.

The use of MPPC allows to improve the signal to noise ratio and therefore the muon detection efficiency. The matrix design is also simplified since clear fibres are no longer necessary. This is a clear advantage during all transportation

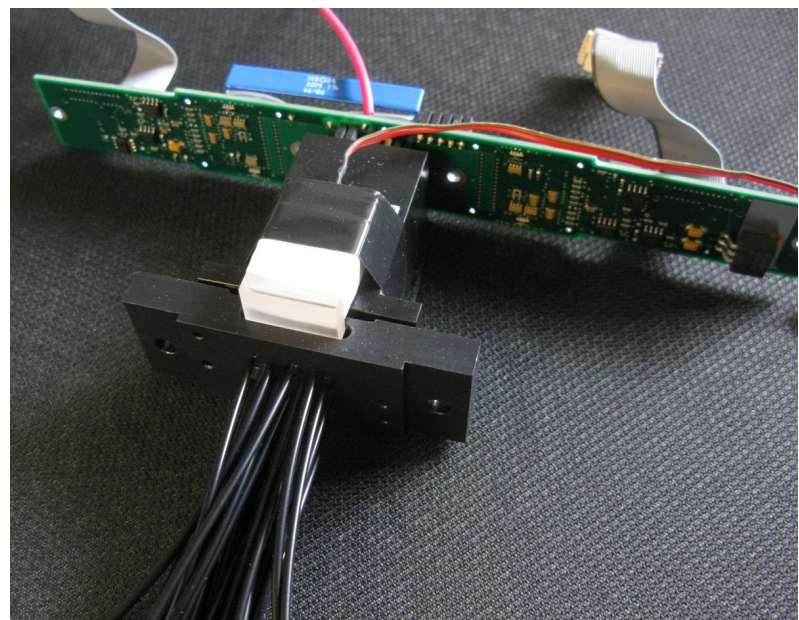

Fig. 3. View of the clear fibres and the cookie fixed in front of the MAPM window. The transparent acrylic piece fixed on the cookie contains a LED used to calibrate the MAPM channels. The frontend board is also visible.

phases where vibrations may damage the fibres or weaken the optical connections. Moreover, no cross-talk is generated at the photomultiplier level. The dark count rate and the thermal fluctuations of the MPPC performances are the most critical features for this type of sensor. While the dark count rate may be controlled by setting a large enough threshold, the thermal fluctuations may be more difficult to reduce in outdoor environments. As an example the dark count rate may double every approximately $9^{\circ} \mathrm{C}$, and high temperature/humidity may affect the device lifetime.

So the main modification to the sensor which has to be performed is the integration of Peltier cells to ensure the temperature stability inside the enclosure containing the MPPCs and a feedback loop in the electronics to finely adjust the bias voltage according to the changes in temperature. The MPPC option is currently under final validation, see for instance the developments in the electronics readout in the next Section. A MPPC matrix is currently used for tests in our laboratories and we foresee to include progressively MPPC matrices in our telescopes, first with MAPM matrices (hybrid option) to compare the performances between the two readout options and then as unique detection device.

\section{Readout system}

\subsection{Data acquisition system}

Each photomultiplier is readout by two multichannel frontend chips, one for each direction $\mathrm{X}$ and $\mathrm{Y}$. They were designed in the AMS BiCMOS $0.8 \mu$ s technology at the Linear Accelerator Laboratory (LAL IN2P3 Orsay, France) for the OPERA experiment (Lucotte et al., 2004). A total time of $12.8 \mu$ s is required to readout a MAPM after reception 


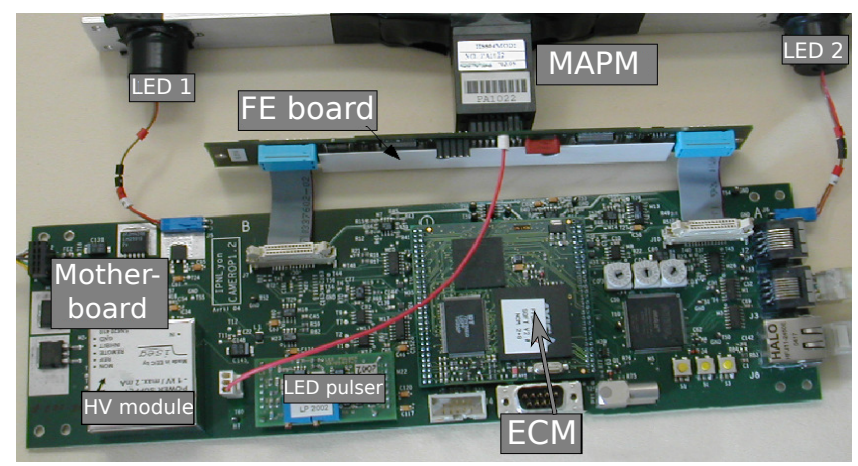

Fig. 4. Readout system for MAPM. The front-end (FE) board, connecting the MAPM, has two multichannel chips for the trigger generation and the signal readout. The mother-board hosts the Ethernet Controller Module (ECM), the High Voltage (HV) module, the LED pulser system and the clock readout devices.

of a trigger, setting the dead-time scale of each telescope plane. The chip consumption is about $150 \mathrm{~mW} \mathrm{pC}^{-1}$ for 32 channels and the electronic cross-talk has been measured to be negligible with respect to optical cross-talk which is around $2 \%$.

The global data acquisition system is built as a network of "smart sensors" (Girerd et al., 2000). Once the data is collected by the multichannel chips, they are digitized and pre-processed by an Ethernet controller module plugged on a controller mother board (Fig. 4). The same type of architecture is also valid for the MPPC option where only the front-end stage has to be adapted. The data acquisition system performs the detector configuration, the monitoring, the event building and data transfer to the on-board computer (Marteau, 2009). The distributed client/server software is based on the Common Object Request Broker Architecture (CORBA) standard (www.corba.org). Each retained hit contains the signal intensity (in ADC counts), the timestamp and the $\mathrm{X}$ and $\mathrm{Y}$ coordinates of the interaction. The readout is running continuously, data are processed and filtered online and stored locally. Since the telescope is running in triggerless mode, hit timestamp accuracy is a critical issue. A clock broadcasting system synchronizes all sensors with a common clock unit regulated by GPS.

\subsection{Clock distribution system}

A global common clock is necessary to synchronize all nodes of the distributed system. The bi-directionality of the system allows the control of the signal reception and the measurement of the propagation time with acknowledgement signals (Marteau, 2009). After correction of the propagation delays, the value of the local fine counters on each sensor is used for the event time stamping, ensuring an overall accuracy of $10 \mathrm{~ns}$, well adapted for defining coincident events between the detection planes during the event building phase. The coincidence window for $\mathrm{XY}$ coincidence is then of $10 \mathrm{~ns}$ at maximum, since it represents one clock shot.

\subsection{On-board computer}

The on-board computer is linked to the Ethernet controller modules and to the WiFi antenna through which it communicates with the outside and acts as a gateway for the whole system. The choice of the hardware was motivated by different considerations and constraints induced by the experiment: its size, field of operation and need of autonomy. The computer has been assembled with off-the-shelf components so as to answer to our needs: simplicity and stability, low consumption, use of market standards.

The on-board computer acts as the server: it runs the data acquisition (DAQ) software that periodically interrogates on a per-cycle basis the sensor applications to retrieve the list of hits: these lists are merged and sorted in time, then, an algorithm is applied, looking for time coincidences and possible clustering logic. Specific cuts on hit position, energy, multiplicity may be applied afterwards. Data are then written directly on hard disk. SQLite, a light data base system is used to handle locally all the configuration and calibration parameters of the whole acquisition system (www.sqlite.org). The DAQ application offers as well a graphical user interface to handle the data acquisition telescope. It offers a full access to all sensors for activation/deactivation, calibration and configuration, and at the telescope level, it allows run start/stop and calibration start/stop.

\subsection{Trigger and readout scheme}

The DAQ system involves two major processes: a sensor application (one per sensor) and a DAQ application (one per telescope) for data collection and event building. The trigger scheme is based on a dual level architecture: Level 0 (L0) applied on the individual plane sensors and Level 1 (L1) applied online by the DAQ mini-PC to the telescope data collected from all planes. The triggering conditions during a standard run are the following:

- L0: at least 1 channel above 1 photo-electron threshold and at least 1 channel above zero suppression threshold (0.2 photo-electron) in the opposite direction (i.e. $1 \mathrm{XY}$ coincidence required in a time of $10 \mathrm{~ns}$ );

- L1: majority condition: at least 2 L0 triggers in time coincidence within a typical $100 \mathrm{~ns}$ gate.

All the triggering parameters stated above are adjustable online by software. 

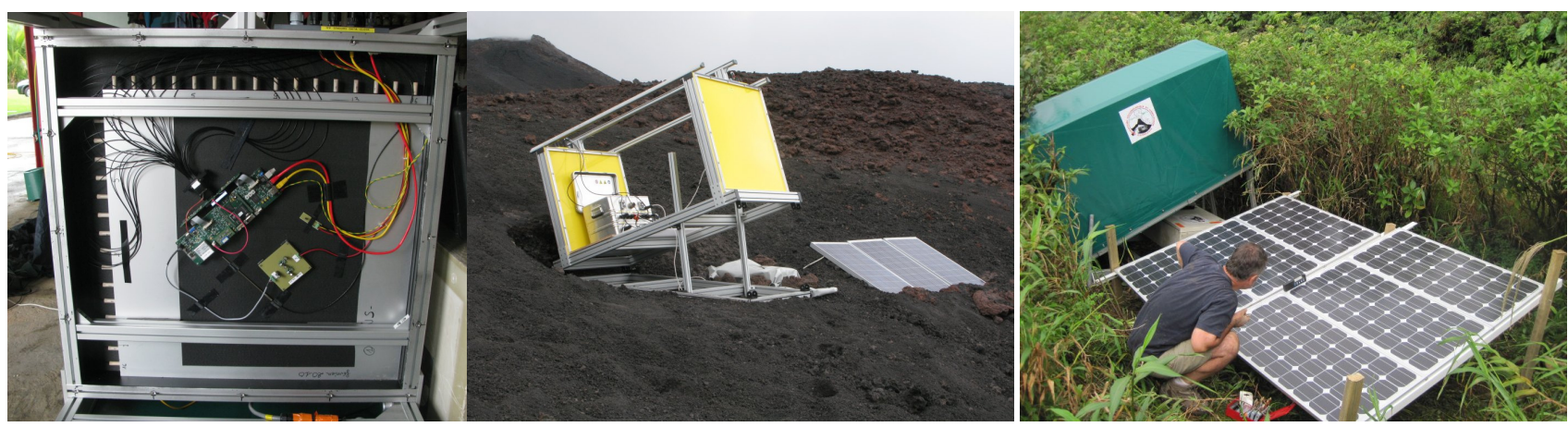

Fig. 5. Left: matrix with open case. Middle: complete telescope at the summit of Mount Etna in Sicily. The dip is $30^{\circ}$ and the distance between the two matrices is of $115 \mathrm{~cm}$. Solar conditions there allow single solar unit use. Right: telescope covered with its tarpaulin on La Soufrière of Guadeloupe. Two solar units of $270 \mathrm{~W}$ each are mandatory because of cloudy weather.

\section{Field telescope}

\subsection{Mechanical structure}

The components described in the preceding Sections were assembled into a robust telescope that can be installed in a harsh environment. Generally, a telescope includes two matrices as shown Fig. 2, but a three-matrices arrangement may be useful to better suppress fake tracks made by two charged particles interacting simultaneously with the two matrices of detection (Nagamine, 2003). In some other case, thick iron plates with a thickness of at least $2 \mathrm{~cm}$ may be necessary to filter electrons (Nagamine, 2003).

The telescope structure must be adaptable to host this optional equipment and to adjust the distance between the matrices in order to adapt the total angular aperture. Moreover the frame must be modular to facilitate both the transportation and the installation on the field. Accounting for these constrains, the frame of the telescope is built with slotted and anodised aluminium profiles.

A scintillator matrix and its devices (cookie, MAPM, front-end, ECM and power supply) are placed in a single box made with 4 profiles and two $1 \mathrm{~mm}$ thick aluminium plates (Fig. 5 left). The interior of the box is painted in black to dump the effects of potential light leaks and the matrix is secured by means of two bars of profile. The total mass of the box amounts to $45 \mathrm{~kg}$ for a $16 \times 16$ pixels matrix and handles are distributed along the frame for easier manipulation. Water and light tightness is obtained with a seal applied between the aluminium plates and the profiles. Four connectors complying with the IP67 norm are used to ensure power supply and data transfer and a valve equipped with a Gore Tex membrane allows evacuation of water vapour without letting liquid water to penetrate into the box. This simple device proved very efficient to reduce the relative humidity in the box, for instance, on la Soufrière, the relative humidity varies around $70 \%$ in the box while it is higher than $95 \%$ outside. We checked that the environmental conditions in the box were always above the dew point.
The supporting structure of the telescope is made with the same aluminium profile used for the matrix boxes (Fig. 5 middle), and it is composed of two rectangular rigid frames and of optional bars to support the tarpaulin (Fig. 5 right). The rectangular frames are articulated to fix the inclination of the matrices, and the lower frame can be either equipped with wheels or with adjustable legs suitable for rough topography. Auxiliary anchors may be placed elsewhere on the frames to fix stabilizing cables. Except for the boxes containing the scintillator matrices, the whole telescope may be disassembled in elements of less than $20 \mathrm{~kg}$.

The common electronic devices (on-board computer, data logger, DC-DC converters, Ethernet switch, clock board) are placed in an aluminium case also equipped with a Gore Tex membrane. This box is hermetically closed against water and dust, and its metallic surface is sufficiently large to ensure good thermal dissipation. In practice, the temperature rarely reaches $45^{\circ} \mathrm{C}$.

\subsection{Environmental sensors}

Several temperature and relative humidity sensors are placed in the matrix and electronic boxes as well as on the telescope frame to monitor the environmental parameters outside, and in the different cases, providing the ambient conditions of the electronics. Two inclinometers are placed on the frame to monitor the orientation of the telescope and check that no catastrophic overturn occurred. The voltage of the power supply is also measured to check the efficiency of the accumulator charged by the solar panels.

All data are collected by a data logger (e.Reader from Gantner, www.bgp.fr) which daily sends its data files to the on-board computer through a ftp connexion. In order to reduce its power consumption to $100 \mathrm{~mW}$, the data logger is almost always in sleep mode and wakes up every $15 \mathrm{~min}$ to perform data logging. 
Table 1. Electrical consumption of the devices.

\begin{tabular}{lr}
\hline Component & Consumption W \\
\hline Acquisition boards & $2 \times 7$ \\
WiFi antenna & 3 \\
On-board computer & 13 \\
Ethernet switch & 2.5 \\
DC/DC converters & 3 \\
Data logger (in wake-up mode) & 0.1 \\
\hline Total & $\approx 36$ \\
\hline
\end{tabular}

\subsection{Electrical power and WiFi link}

The overall power consumption of the telescope is of about $35 \mathrm{~W}$ (Table 1) and allows the use of solar units to provide electrical energy. Eventually, in an environment where volcanic ash may alter the solar panels, combustible cells may be used as well. The total power of solar units to be installed depends on the meteorological conditions encountered on field. For instance, the cloudy weather generally present on La Soufrière of Guadeloupe implies an application of a security factor of at least 15 to prevent from power failure during long cloudy periods, where the solar panels are not efficient enough to refill the accumulators. For this reason, on this volcano we use two solar units with a maximum power capacity of about $540 \mathrm{~W}$ (Fig. 5 right). WiFi antennas are used to establish a permanent link with the telescope in order to both transfer data and perform a real time monitoring.

\section{Calibration of the telescope response}

\subsection{Photomultiplier calibration}

A first calibration concerns the equalization of the MAPM channels in order to work with a single threshold for all channels and therefore simplify the trigger logic. A blue-light LED diffusing system is plugged into the cookie (Fig. 3) to calibrate the MAPM response by a statistical analysis of each MAPM channel spectra, fitted with a Bellamy model which leads to an average $10 \%$ accuracy on the parameters value (Bellamy et al., 1994). The LED system is used both for calibrating each MAPM, as described in the following, and for monitoring the system stability in-situ.

The MAPM response to a light pulse results from both the detection of the emitted photons and the signal amplification. The calibration procedure of a MAPM proceeds as follows:

1. LED spectra fitting at $\mathrm{HV}=-850 \mathrm{~V}$ with $1-2$ photoelectrons injected on average,

2. identification of the channel(s) with the maximal response (gain),
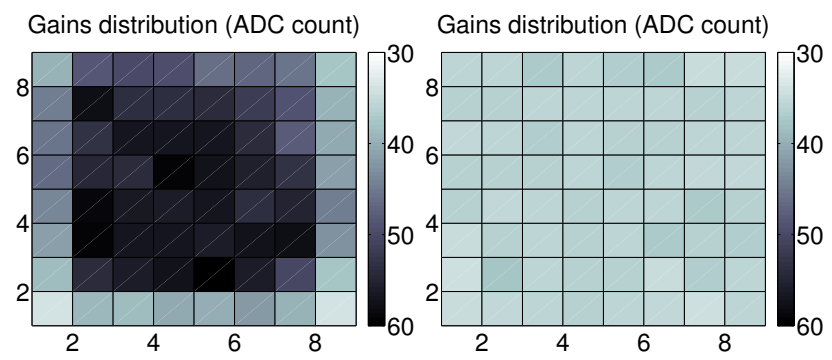

Fig. 6. Gains distribution over the $8 \times 8$ matrix before (left) and after (right) gain correction.

3. first raw correction of the high voltage to fix the maximal response at $10^{6}$ using a scaling law such as the gain is set proportional to a power law of the high voltage,

4. first determination of the MAPM response parameters to a pulse light as described in Bellamy et al. (1994),

5. equalization of the 64 channels gains and insertion of the shift register parameters in the front-end electronics,

6. LED spectra fitting in this new configuration,

7. second iteration of the procedure if necessary,

8. threshold setting around 0.1 photo-electron, measurement of the dark count rate in auto-triggering mode.

The gain distributions of the 64 MAPM channels before and after the gain equalisation are shown in Fig. 6.

Finally, the MAPM is connected to the telescope plane and the counting rate is measured in auto-triggering mode. As the counting rate includes the natural radioactivity and the MAPM dark current of the isolated channels as well as the cosmics rate $\left(\sim 0.01 \mathrm{~cm}^{-2} \mathrm{sr}^{-1} \mathrm{~s}^{-1}\right)$, the software is configured to select only $\mathrm{X}$ and $\mathrm{Y}$ coincidences (L0 trigger). On field, the trigger rate per channel ranges between 10 and $25 \mathrm{~Hz}$. When the L1 trigger is applied (at the telescope level) this trigger rate decreases between 0.27 and $1.67 \mathrm{~Hz}$.

When the photomultipliers of each telescope plane are calibrated, a dedicated cosmic run is started with the planes kept horizontal. The event selection is limited to the most vertical tracks which correspond to particles with a trajectory perpendicular to the planes of detection. The response of the telescope plane to the minimum ionising particles is measured using these vertical events and recorded for future offline data-correction.

The total cross-talk is then measured, it includes all possible sources: electronics, MAPM (coupling between anodes on the input window) and cookie-to-MAPM optical coupling. After alignment of the MAPM on the cookie, $8 \%$ of the total light signal sent on a pixel is shared by the neighbouring channels. Each MAPM pixel has 8 neighbours: 4 with a common side (each receiving about $2 \%$ on the total light) and 4 at the corner which have a negligible role. 
For instance, in the $16 \times 16$ matrices the clear fibres are connected only every two MAPM pixels so any confusion between cross-talk and multiple events is avoided. In field measurements, despite possible misalignments caused by the transportation vibrations, $3.25 \%$ of the total light detected by a pixel is seen by its neighbours.

\subsection{Acceptance of the telescope}

The trajectory of a detected event is given by the $\mathrm{X}(i, k)$ and $\mathrm{Y}(j, l)$ coordinates of the two fired pixels belonging to the front $\left(P_{i, j}^{\mathrm{F}}\right)$ and rear $\left(P_{k, l}^{\mathrm{R}}\right)$ matrices of detection. All pairs of pixels $\left\{P_{i, j}^{\mathrm{F}}, P_{k, l}^{\mathrm{R}}\right\}$, with the same relative position $\{m=i-k, n=j-l\}$, share the same average direction $\mathbf{r}_{m, n}$. The number of muons detected by the telescope for a given direction $\mathbf{r}_{m, n}$ reads,

$N\left(\mathbf{r}_{m, n}, \Delta T\right)=I\left(\mathbf{r}_{m, n}\right) \times \Delta T \times \mathcal{T}\left(\mathbf{r}_{m, n}\right)$,

where $I$ is the flux of muons given in $\mathrm{cm}^{-2} \mathrm{sr}^{-1} \mathrm{~s}^{-1}, \Delta T$ is the duration of the measurement period, and $\mathcal{T}$ is the telescope acceptance expressed in $\mathrm{cm}^{2} \mathrm{sr}$. The acceptance may be written as,

$\mathcal{T}\left(\mathbf{r}_{m, n}\right)=S\left(\mathbf{r}_{m, n}\right) \times \delta \Omega\left(\mathbf{r}_{m, n}\right)$,

where $S$ is the detection surface and $\delta \Omega$ is the angular aperture. For a given direction $\mathbf{r}_{m, n}, S$ is controlled by the pixel size $d$ and by the number of pairs of pixels having a same $\{m, n\}$, and $\delta \Omega$ depends on the distance $D$ between the matrices and on the distances $x_{\text {shift }}$ and $y_{\text {shift }}$ corresponding to $m$ and $n$. Figure 7 shows the angular aperture and the acceptance for the $\left(2 N_{x}-1\right) \times\left(2 N_{y}-1\right)=961$ discrete directions $\mathbf{r}_{m, n}$ of a telescope with two $16 \times 16$ matrices separated by $D=115 \mathrm{~cm}$, as this shown in Fig. 5. As expected, the acceptance is maximal for the direction $\mathbf{r}_{0,0}$, perpendicular to the matrices, since all pixels contribute to the detection surface which is then maximum. The acceptance is small for a margin corresponding to the directions that most depart from $\mathbf{r}_{0,0}$ and only a fraction of all possible directions of detection will be efficient in practice. With $D=115 \mathrm{~cm}$ and for the $\mathbf{r}_{0,0}$ direction, the solid angle is about $2 \times 10^{-3} \mathrm{sr}$, allowing to detect heterogeneities with a size of about $10 \mathrm{~m}$ at a distance of $500 \mathrm{~m}$, in a few months. To reduce the detection time, two options increasing the solid angles can be considered: a reduction of the distance between the matrices or a merging of some angles of view in a post-processing to increase the solid angle of detection, both options affect the telescope resolution.

The actual acceptance of a telescope also depends on the efficiency of the scintillator bars forming the matrices. Depending on the quality of optical couplings, a scintillator bar may have a low response (see for example bar Y12 of the rear matrix, Fig. 8). This failure may be caused by a bad connection between optical fibres generated during transportation.
Having the number $N$ of muons detected for each direction $\mathbf{r}_{m, n}$ during a period $\Delta T$, the integrated flux $I$ reads,

$I\left(\mathbf{r}_{m, n}\right)=\frac{N\left(\mathbf{r}_{m, n}\right)}{\Delta T \times \mathcal{T}\left(\mathbf{r}_{m, n}\right)}\left(\mathrm{cm}^{-2} \mathrm{sr}^{-1} \mathrm{~s}^{-1}\right)$.

The flux computed with the theoretical acceptance of the telescope (Fig. 7 middle) is shown on the top part of Fig. 9. As expected, this open sky flux varies principally with the zenith angle (Barrett et al., 1952) from $1 \times 10^{-4}$ to $8.5 \times$ $10^{-3} \mathrm{~cm}^{-2} \mathrm{sr}^{-1} \mathrm{~s}^{-1}$, but one can observe several defects affecting the expected circular symmetry originated by the muon flux invariance with azimuth angles. These defects disappear if the acceptance model takes into account the Y12 bar failure, as shown in the right part of Fig. 7. The corrected muon flux recover its circular symmetry around the zenith as can be observed on the bottom part of Fig. 9. A more accurate calibration method of this sensor has been developed to correct any image distortion due to more or less efficient bars, see Lesparre et al. (2012).

\section{First field measurements}

The installation of a muon telescope on field with harsh conditions requires first runs of calibration to ensure the sensor efficiency. We check the fluctuations with time of the total number of events detected and their distribution per bar to detect any loss in efficiency of the whole telescope or any bar failure (Fig. 8). The photomultipliers show the same gain and pedestal stabilities as in laboratory. The cross-talk measured on field did not exhibit any changes despite vibrations during the transportation leading to possible changes in the cookie-MAPM alignment.

Four telescopes with $N_{x}=N_{y}=16$ matrices are still operating in various field and weather conditions (Fig. 5): Mont-Terri underground laboratory, summit of Mount Etna in Sicily, Soufrière of Guadeloupe, Britanny in France (Marteau et al., 2011). We recorded consistent data sets to establish first radiographies of Mount Etna and Soufrière of Guadeloupe, besides series of measurement in the Mont Terri underground laboratory (Switzerland) will give us an access to establish a 3-D radiography with trajectories intersecting inside the solid rock. Compared to the data set acquired in open-sky conditions, the muon flux decreases strongly when the sensor is configured to image a geological object. For instance when the sensor is placed inside the Mont Terri underground laboratory, the detected flux is of $3 \times 10^{-6} \mathrm{~cm}^{-2} \mathrm{sr}^{-1} \mathrm{~s}^{-1}$ on average for a thickness of rock sounded of about $400 \mathrm{~m}$. Across La Soufrière de Guadeloupe volcano the detected flux may be lower than $1 \times$ $10^{-6} \mathrm{~cm}^{-2} \mathrm{sr}^{-1} \mathrm{~s}^{-1}$ as the thickness of rock reaches $800 \mathrm{~m}$ for some angles of view of the telescope. The detected flux is thus severely reduced by the presence of hundreds meters of rock in between the sensor and the incoming cosmic muons. In such conditions it is important to consider the 

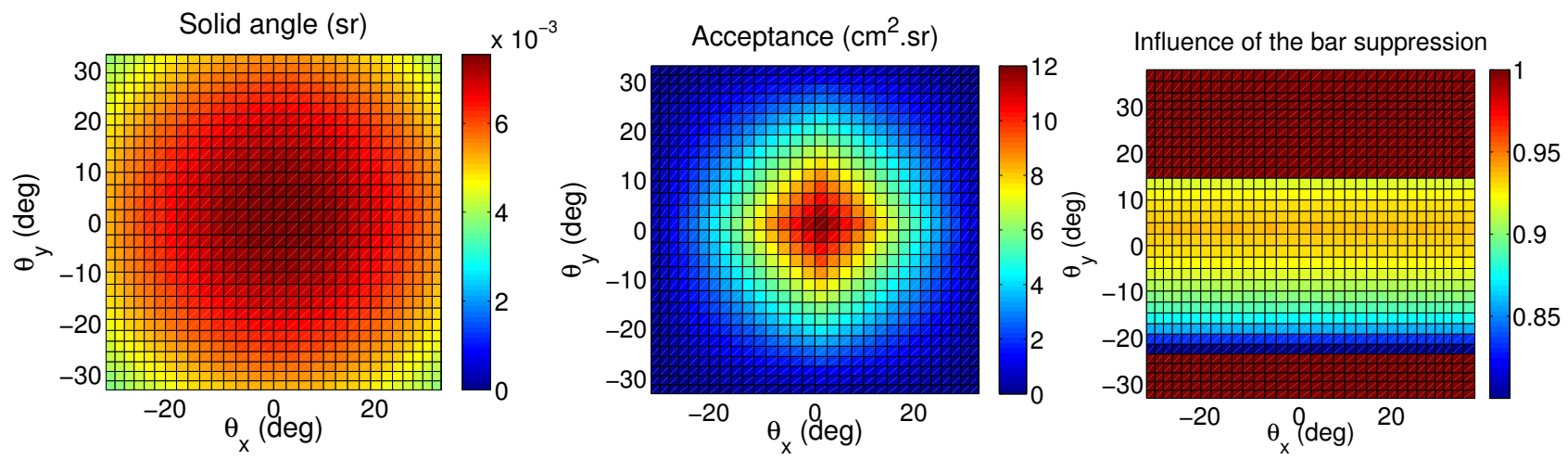

Fig. 7. Azimuthal angular properties of a telescope equipped with two $16 \times 16$ matrices with pixel size $d=5 \mathrm{~cm}$ and separated by $D=$ $115 \mathrm{~cm}$. Left: angular resolution $\delta \Omega$ for each discrete direction $\mathbf{r}_{m, n}$. Middle: acceptance $\mathcal{T}\left(\mathbf{r}_{m, n}\right)$. Right: ratio of the corrected acceptance taking into account for the dysfunction of the bar Y12 of the rear matrix (Fig. 8) on the theoretical acceptance computed with all bars.
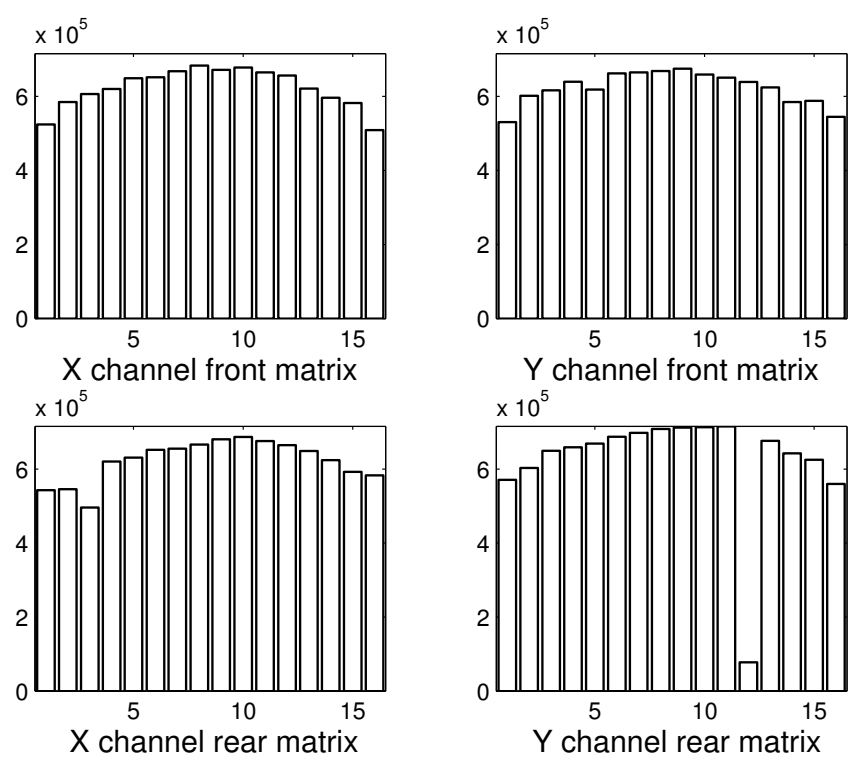

Fig. 8. Events distribution detected on the front and rear $\mathrm{X}$ and $\mathrm{Y}$ planes. The data are collected during $170 \mathrm{~h}$ under a zenith angle of $1.35^{\circ}$ and for an azimuth angle of $45^{\circ}$.

level of background noise and perform means to reduce its amplitude such as the installation of a third detection matrix or absorbing screens (Nagamine, 2003).

The telescope installed on La Soufrière has now more than one year of measurements without any failure despite the hurricane season, strong wind occurrence and heavy rain episodes exceeding $100 \mathrm{~mm} \mathrm{day}^{-1}$. The monitoring of this telescope allows to check that the relative humidity inside the electronic containers varies around $70 \%$ and that the dew point is far from being reached. Because of cloudy periods which may last a full week, the acquisition may be switched off because of power failure. A WiFi link allows the monitoring of the acquisitions interruption and relaunch. A three-planes detection configuration is used and only events

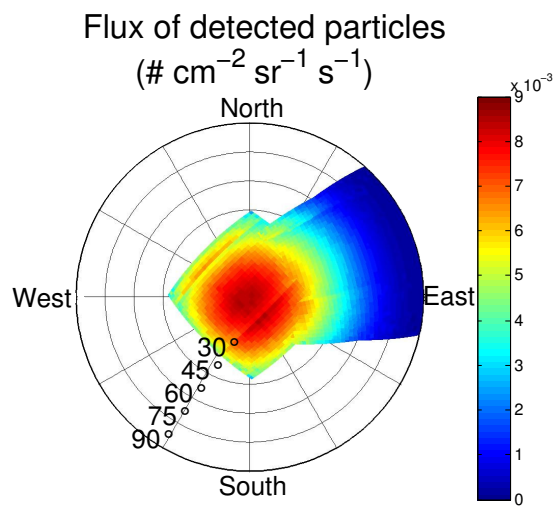

Corrected flux of detected particles

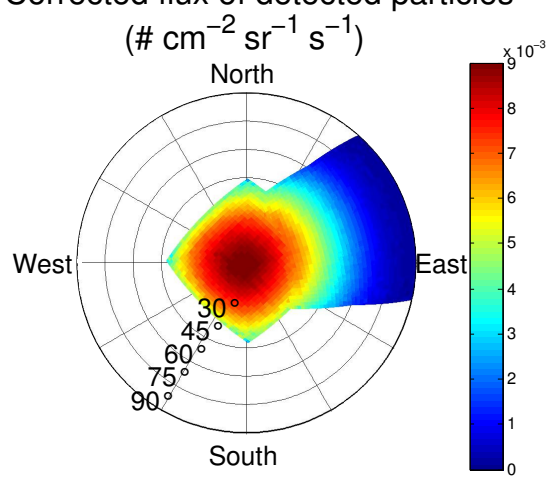

Fig. 9. Top: detected flux in open sky conditions. Number of particles detected divided by the acquisition time and the theoretical telescope acceptance. Bottom: corrected flux of particles detected, computed with the acceptance on right Fig. 7. The zenith angles are indicated.

in triple coincidence with a straight trajectory are retained. The presence of the third detection plane increases the purity of the data sample by filtering fake tracks, which may bias the analysis of the muon flux attenuation through matter (Nagamine, 2003). Analysis of current and past data samples is still underway while statistics are increasing and new 
actions are planned to better constrain our results (new open sky calibration runs in triple coincidence and completion of a Geant4 simulation model).

\section{Conclusions and perspectives}

In this article we detail the design, commissioning and running of scintillator telescopes dedicated to muon tomography of large geological structures such as volcanoes. The telescopes architecture is adapted from a well-known technology used in large high energy physics experiments such as OPERA (Lucotte et al., 2004; Marteau et al., 2011).

Data acquisition are now underway on different field conditions while the data analysis is under development. A calibration method to correct bars different efficiencies has been established (Lesparre et al., 2012). The telescope commissioning and running protocols are validated.

We propose the MPPC option to replace the MAPMs. Tests and comparisons are underway to produce quickly a new hybrid telescope mixing both photosensors. As the telescope set up is confirmed, bigger matrices with $24 \times 32$ pixels are in construction to improve the telescope acceptance. Each telescope developed for an installation in open sky will be equipped with at least three matrices in order to increase the signal to noise ratio, and therefore the images contrast. After the completion of the exploratory steps detailed in the present article, a very rich and promising programme of data taking, research and development as well as modelization are now established.

Acknowledgements. The design and the construction of the telescopes greatly benefited from the skilled expertise of Karim Mahiouz, Franck Mounier and Pascal Rolland (mechanical engineering) and Sylvain Vanzetto (optical fibres). Optical plugs are designed and machined by Troimeca, and the tarpaulins are manufactured by Tigier. Daniele Carbone, Sylvain Pasquet and Justine Restout participated to the construction of the telescopes. The field installation on La Soufrière de Guadeloupe benefited from the generous help of the Observatoire Volcanologique et Sismologique de Guadeloupe staff, particularly from: Jean-Bernard De Chabalier, Alexis Bosson, Frédéric Randriamora, Thierry Kitou, Christian Lambert and Véronique Daniel. We thank both referees for their constructive reviews. The DIAPHANE project is financially supported by the IPGP (www.ipgp.fr) BQR grant, the DOMOSCAN ANR project (http://www.agence-nationale-recherche.fr), the CNRS/IN2P3 (www.cnrs.fr) Astroparticles program, and the MD experiment of the Mont Terri project (http://www.mont-terri.ch) funded by Swisstopo and CRIEPI partners. This is IPGP contribution 3247.

Edited by: A. Benedetto

\section{References}

Adam, T., Baussan, E., Borer, K., Campagne, J. E., Chon-Sen, N., De La Taille, C., Dick, N., Dracos, M., Gaudiot, G., Goeltzenlichter, T., Gornushkin, Y., Grapton, J.-N., Guyonnet, J.-L., Hess, M., Igersheim, R., Janicsko Csathy, J., Jollet, C., Juget, F., Kocher, H., Krasnoperov, A., Krumstein, Z., Martin-Chassard, G., Moser, U., Nozdrin, A., Olchevski, A., Porokhovoi, S., Raux, L., Sadovski, A., Schuler, J., Schütz, H.-U., Schwab, C., Smolnikov, A., Van Beek, G., Vilain, P., Wälchli, T., Wilquet, G., and Wurtz, J.: The OPERA experiment target tracker, Nucl. Instrum. Meth. A, 577, 523-539, doi:10.1016/j.nima.2007.04.147, 2007.

Alvarez, L. W., Anderson, J. A., Bedwei, F. E., Burkhard, J., Fakhry, A., Girgis, A., Goneid, A., Hassan, F., Iverson, D., and Lynch, G.: Search for hidden chambers in the pyramids, Science, 167, 832-839, 1970.

Barrett, P., Bollinger, L. M., Cocconi, G., Eisenberg, Y., and Greisen, K.: Interpretation of cosmic-ray measurements far underground, Rev. Mod. Phys., 24, 133-178, 1952.

Bellamy, E. H., Bellettini, G., Budagov, J., Cervelli, F., ChirikovZorin, I., Incagli, M., Lucchesi, D., Pagliarone, C., Tokar, S., and Zetti, F.: Absolute calibration and monitoring of a spectrometric channel using a photomultiplier, Nucl. Instrum. Meth. A, 339, 468-476, doi:10.1016/0168-9002(94)90183-X, 1994.

De Asmundis, R., Avella, P., and Toglia, F.: Using RPC detectors as cosmic rays monitor, IEEE T. Nucl. Sci., 54, 670-676, doi:10.1109/TNS.2007.895505, 2007.

Gaisser, T. K. and Stanev, T.: Cosmic rays, in: Review of particle properties, edited by: Alvarez-Gaumé, L., Blaizot, J. P., Cvetic, M., Doser, M., Geesaman, D. F., Giudice, G. F., Grinstein, B., Haxton, W., Metag, V., Ringwald, A., Rolandi, L., Schlatter, W. D., Weerts, H., and Yanagida, T., Phys. Rev. Lett., 667, 254-260, doi:10.1016/j.physletb.2008.07.028, 2008.

Gibert, D., Beauducel, F., Déclais, Y., Lesparre, N., Marteau, J., Nicollin, F., and Tarantola, A.: Muon tomography: plans for observations in the lesser antilles, Earth Planets Space, 62, 153165, doi:10.5047/eps.2009.07.003, 2010.

Giomataris, I., De Oliveira, R., Andriamonje, S., Aune, S., Charpak, G., Colas, P., Fanourakis, G., Ferrer, E., Giganon, A., Rebourgeard, P., and Salin, P.: Micromegas in a bulk, Nucl. Instrum. Meth. A, 560, 405-408, doi:10.1016/j.nima.2005.12.222, 2006.

Girerd, C., Gardien, S., Burch, J., Katsanevas, S., and Marteau, J.: Ethernet network-based DAQ and smart sensors for the OPERA long-baseline neutrino experiment, in: IEEE Nucl. Sci. Conf. R., Lyon, France, 15-20 October 2000, 2, 12/111-12/115, doi:10.1109/NSSMIC.2000.949950, 2000.

Lesparre, N., Gibert, D., Marteau, J., Déclais, Y., Carbone, D., and Galichet, E.: Geophysical muon imaging: feasibility and limits, Geophys. J. Int., 183, 1348-1361, doi:10.1111/j.1365246X.2010.04790.x, 2010.

Lesparre, N., Gibert, D., and Marteau, J.: Bayesian dual inversion of experimental telescope acceptance and integrated flux for geophysical muon tomography, Geophys. J. Int., 188, 490-497, doi:10.1111/j.1365-246X.2011.05268.x, 2012.

Lucotte, A., Bondil, S., Borer, K., Campagne, J. E., Cazes, A., Hess, M., De La Taille, C., Martin-Chassard, G., Raux, L., and Repellin, J. P.: A front-end readout chip for the OPERA scintillator tracker, Nucl. Instrum. Meth. A, 521, 378-392, doi:10.1016/j.nima.2003.10.104, 2004. 
Malmqvist, L., Jonsson, G., Kristiansson, K., and Jacobsson, L.: Theoretical studies of in-situ rock density determination using cosmic-ray muon intensity measurements with application in mining geophysics, Geophysics, 44, 1549, doi:10.1190/1.1441026, 1979.

Marteau, J.: The OPERA global readout and GPS distribution system, Nucl. Instrum. Meth. A, 617, 291-293, doi:10.1016/j.nima.2009.10.095, 2010.

Marteau, J., Gibert, D., Lesparre, N., Nicollin, F., Noli, P., and Giacoppo, F.: Muons tomography applied to geosciences and volcanology, Nucl. Instrum. Meth. A, doi:10.1016/j.nima.2011.11.061, in press, 2011.

Mohammad-Djafari, A. and Dinten, J. M.: Tomographic reconstruction from few projections, in: Bayesian approach to inverse problems, digital signal and image processing series, edited by: Idier, J., ISTE Ltd and John Wiley \& Sons In, London, United Kingdom, 307-334, 2008.

Nagamine, K.: Geo-tomographic observation of inner-structure of volcano with cosmic-ray muons, J. Geogr., 104, 998-1007, 1995.

Nagamine, K.: Introductory to muon science, Cambridge university press, New York, United States of America, 2003.

Nagamine, K., Iwasaki, M., Shimomura, K., and Ishida K.: Method of probing inner-structure of geophysical substance with the horizontal cosmic-ray muons and possible application to volcanic eruption prediction, Nucl. Instrum. Meth. A, 356, 585-595, doi:10.1016/0168-9002(94)01169-9, 1995.

Pla-Dalmau, A., Bross, A. D., and Mellott, K. L.: Low-cost extruded plastic scintillator, Nucl. Instrum. Meth. A, 466, 482-491, doi:10.1016/S0168-9002(01)00177-2, 2001.
Tanaka, H. K. M., Nagamine, K., Kawamura, N., Nakamura, S. N., Ishida, K., and Shimomura, K.: Development of a two-fold segmented detection system for near horizontally cosmic-ray muons to probe the internal structure of a volcano, Nucl. Instr. Meth. A, 507, 657-669, doi:10.1016/S0168-9002(03)01372-X, 2003.

Tanaka, H. K. M., Nagamine, K., Nakamura, S. N., and Ishida, K.: Radiographic measurements of the internal structure of Mt. West Iwate with near-horizontal cosmic-ray muons and future developments, Nucl. Instrum. Meth. A, 555, 164-172, doi:10.1016/j.nima.2005.08.099, 2005.

Tanaka, H. K. M., Nakano, T., Takahashi, S., Yoshida, J., and Niwa, K.: Development of an emulsion imaging system for cosmic-ray muon radiography to explore the internal structure of a volcano, Mt. Asama, Nucl. Instrum. Meth. A, 575, 489-497, doi:10.1016/j.nima.2007.02.104, 2007.

Tanaka, H. K. M., Uchida, T., Tanaka, M., Takeo, M., Oikawa, J., Ohminato, T., Aoki, Y., Koyama, E., and Tsuji, H.: Detecting a mass change inside a volcano by cosmic-ray muon radiography (muography): First results from measurements at Asama volcano, Japan, Geophys. Res. Lett., 36, L17302, doi:10.1029/2009GL039448, 2009.

Tanaka, H. K. M., Taira, H., Uchida, T., Tanaka, M.,Takeo, M., Ohminato, T., Aoki, Y., Nishitama, R., Shoji, D., and Tsuiji, H.: Three-dimensional computational axial tomography scan of a volcano with cosmic ray muon radiography, J. Geophys. Res., 115, B12332, doi:10.1029/2010JB007677, 2010. 